\title{
Prise en compte du long terme et modalités de gestion des déchets radioactifs
}

\author{
C. SCHIEBER ${ }^{1}$, T. SCHNEIDER ${ }^{1}$, S. LAVELLE ${ }^{2}$
}

(Manuscrit reçu le 2 août 2007, accepté le 10 novembre 2007)

RÉSUMÉ Les déchets radioactifs introduisent une dimension temporelle sans précédent dans le domaine de la gestion des risques. C'est pourquoi, depuis plus de dix ans, des réflexions ont été initiées sur les dispositifs sociétaux et organisationnels permettant d'assurer une prise en charge responsable du risque associé aux déchets radioactifs sur le long terme. Ces réflexions conduisent à s'interroger sur les interactions entre les enjeux sociétaux et les critères de radioprotection, faisant ainsi appel à une approche multidisciplinaire. Dans le cadre du projet européen COWAM 2, dédié à l'amélioration de la gouvernance de la gestion des déchets radioactifs en Europe, un groupe de travail impliquant des experts, des autorités de sûreté et de radioprotection, des gestionnaires de déchets, des élus locaux et des associatifs, a discuté des enjeux associés à la prise en compte du long terme. Cet article présente les principaux résultats de ce groupe de travail, organisés autour de quatre thèmes : la signification et les enjeux du long terme, les enjeux éthiques associés à la dimension du long terme, la continuité et la durabilité de la surveillance et du contrôle des installations recevant des déchets radioactifs, l'efficacité des dispositifs de financement de la gestion des déchets radioactifs sur le long terme.

ABSTRACT Taking into account the long term dimension associated with radioactive waste management.

Radioactive waste introduces a new time dimension in the field of risk management. This is why, for more than $\mathbf{1 0}$ years, there have been reflections on the societal and organisational mechanisms allowing a responsible management over the long term of the risk associated with radioactive waste. These reflections lead one to ask questions regarding interactions between what is at stake for societal and radiation protection criteria, demanding a multidisciplinary approach to the problem. Within the framework of the European project COWAM 2, dedicated to the improvement of governance of radioactive waste management in Europe, a working group involving experts, authorities, waste managers, locally elected representatives and NGOs, discussed the stakes associated with the long term dimension. This article presents the main results of this working group, organised around four themes: meaning of the long term and what is at stake, the ethical dimension regarding long term issues, continuity and sustainability of the surveillance and control of radioactive waste facilities, effectiveness of financing schemes for the long term management of radioactive waste.

Keywords: waste management / long term / ethics

1 Centre d'étude sur l'évaluation de la protection dans le domaine nucléaire (CEPN), 28 rue de la Redoute, 92260 Fontenayaux-Roses, France.

2 Institut catholique d'arts et métiers (ICAM), 6 rue Auber, 59046 Lille, France. 


\section{Introduction}

Du fait de la période radioactive de certains radionucléides, de leur nature chimique et de leur concentration, les déchets radioactifs ont la particularité d'introduire une dimension temporelle qui n'a jamais été expérimentée jusqu'à présent dans les dispositifs de gestion des risques. Selon les déchets, compte tenu de la période radioactive, il peut s'agir de prendre en considération des échelles de temps de l'ordre de plusieurs milliers, voire millions d'années. Afin de pouvoir élaborer des dispositifs de gestion adaptés à cette situation, des réflexions ont été initiées depuis plus de dix ans sur les enjeux sociétaux associés à la gestion des déchets radioactifs (AEN/OCDE, 1995 ; Hériard-Dubreuil et al., 2003, 1998, 1996 ; Lavelle, 2006). Ces réflexions ont notamment mis en évidence que, du point de vue sociétal, la prise en compte du temps soulève essentiellement la question des modalités de transfert des déchets radioactifs à la génération suivante, puis aux générations futures. Ces modalités concernent non seulement le transfert des dispositifs de protection mais aussi les moyens à mettre en œuvre pour assurer une continuité de la surveillance sur le long terme. Ainsi, afin d'assurer une mise en œuvre efficace de la radioprotection, il convient à la fois de développer des indicateurs pour évaluer la performance des systèmes techniques et géologiques sur le long terme et de s'interroger sur les dispositifs d'accompagnement qui permettront le transfert du système de protection au cours du temps.

Dans le cadre du projet européen COWAM $2^{3}$ dédié à l'amélioration de la gouvernance de la gestion des déchets radioactifs en Europe (Hériard-Dubreuil et al., 2007), un groupe de travail, comprenant une vingtaine de personnes (experts, autorités, gestionnaires de déchets, élus locaux et associatifs), a discuté, sur la base de travaux de recherche et d'expériences, des enjeux et des modalités de gestion associés à la prise en compte du long terme ${ }^{4}$. Le mandat de ce groupe de travail était d'identifier, de discuter et d'analyser les aspects institutionnels, éthiques, économiques et juridiques associés à la présence sur le long terme d'un site de stockage ou d'entreposage de déchets. L'objectif était de proposer des recommandations pour une meilleure prise en compte des problèmes de long terme dans les processus de prise de décision et dans les modalités de gestion des déchets (Schneider et al., 2006). Cet article synthétise les principaux développements et conclusions de ce groupe de travail, autour des quatre thèmes suivants :

- la signification et les enjeux du long terme et des générations futures dans le cadre de la gestion des déchets radioactifs,

\footnotetext{
3 Le projet européen COWAM 2 sur la gouvernance de la gestion des déchets radioactifs, coordonné par Mutadis Consultants, s'est déroulé de 2004 à 2006 (voir Annexe 1).

4 La liste des membres du groupe de travail sur la prise en compte du long terme dans les modalités de gestion des déchets radioactifs est présentée en Annexe 2.
} 
- les enjeux éthiques associés à la dimension du long terme pour la gestion des déchets radioactifs,

- la continuité et la durabilité de la surveillance et du contrôle des installations recevant des déchets radioactifs,

- l'efficacité des dispositifs de financement de la gestion des déchets radioactifs sur le long terme.

\section{Signification et enjeux du « long terme »}

Il n'existe pas de définition «unique » du long terme et différentes échelles de temps peuvent être considérées selon les enjeux et les perspectives temporelles des acteurs qui considèrent cette question. En ce qui concerne la gestion des déchets radioactifs, deux principaux points de vue peuvent être distingués : le point de vue technique et le point de vue sociétal.

\subsection{Le long terme du point de vue technique}

Du point de vue technique, le long terme est généralement considéré par les exploitants des installations de gestion des déchets et par les autorités de sûreté et de radioprotection dans le cadre de l'évaluation de la performance des systèmes de protection sur des périodes de temps de l'ordre de plusieurs milliers d'années, voire plus (jusqu'à quelques millions d'années). Les critères d'évaluation de la sûreté $^{5}$ des stockages sont en effet établis en fonction de l'évolution de la radioactivité et de la température des colis de déchets, de la durée de vie des installations et des colis de déchets, des évolutions géologiques et de l'évaluation des impacts des stockages sur l'environnement.

Dans cette perspective, il est utile de rappeler les ordres de grandeur associés à ces évolutions temporelles. Les paragraphes ci-après illustrent l'évolution de l'activité d'un colis de déchets vitrifiés et de son débit de dose.

Ainsi, à la sortie de l'opération de vitrification, un colis de déchets vitrifiés d'une capacité de 150 litres de produits hautement radioactifs contient de l'ordre de (Lagrange, 2005) :

- $100 \mathrm{MBq}$ pour les divers isotopes d'uraniums,

- $1 \mathrm{TBq}$ des divers isotopes de plutoniums,

- $100 \mathrm{TBq}$ d'américium et de curium, que l'on appelle aussi les actinides mineurs,

- $10000 \mathrm{TBq}$ de produits de fission, tels que le ruthénium, le strontium et le césium.

5 Dans le domaine de la gestion des déchets radioactifs, le terme sûreté intègre la dimension radioprotection. 


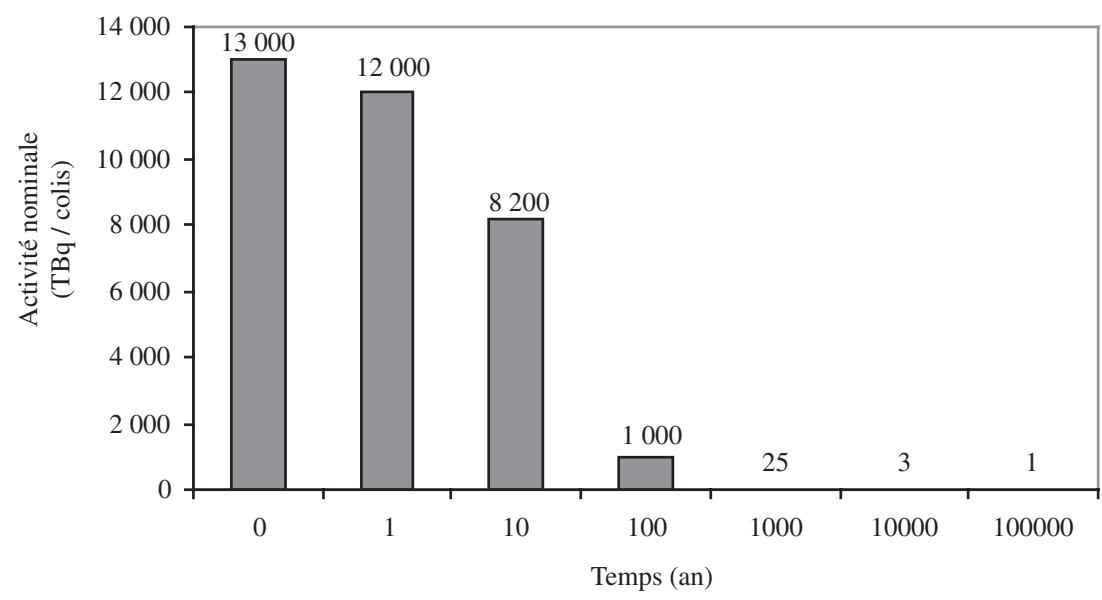

Figure 1 - Évolution de l'inventaire radiologique nominal d'un colis de déchets vitrifiés (colis type C1). Evolution of the nominal activity of a vitrified waste package (C1 type).

\subsubsection{L'évolution de l'activité}

L'évolution de l'activité dépend des radionucléides présents dans les colis de déchets et de leur période radioactive. Ainsi, l'activité nominale des isotopes de plutonium est divisée par environ 20 en 100 ans, par 60 en 1000 ans et par 200 en 10000 ans. L'activité des actinides mineurs est divisée par environ 4 en 100 ans, par 20 en 1000 ans et par 1000 en 10000 ans. L'activité de l'uranium diminue très peu. Pour les produits de fission à vie courte (de l'ordre de 30 ans comme par exemple le strontium-90 et le césium-137), l'activité est divisée par environ 10 en 100 ans, par $10^{10}$ en 1000 ans et près de $2 \times 10^{100}$ après 10000 ans. Par contre, pour les produits de fission à vie longue (comme le technicium-99 dont la demievie est d'un million d'année), il faut plusieurs centaines de milliers d'années pour obtenir une décroissance significative de leur activité.

La figure 1 synthétise de façon simplifiée - en sommant l'activité de l'ensemble des radionucléides présents - l'évolution de l'activité d'un colis de déchets vitrifiés (exprimée en TBq/colis) (Lagrange, 2005).

\subsubsection{L'évolution du débit de dose}

Du fait de la décroissance de l'activité des différents radionucléides présents dans les colis de déchets vitrifiés, le débit de dose des colis décroit rapidement. Il est divisé par près de 10 après une période de 100 ans et par 100000 après une période 


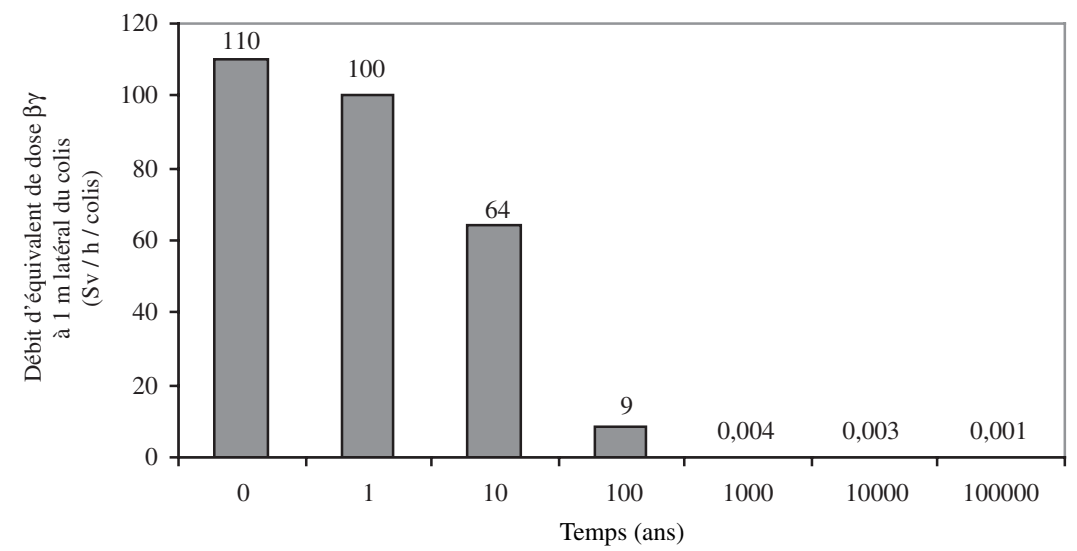

Figure 2 - Évolution du débit d'équivalent de dose à un mètre en latéral d'un colis de déchets vitrifiés (colis type C1).

Evolution of the dose rate at one meter lateral from a vitrified waste package (C1 type).

de 1000 ans. Au-delà de cette période, la réduction devient limitée. Ainsi, après une période de 100 ans, une exposition de plusieurs heures, sans protection et à une distance d'un mètre d'un colis, entraînerait une dose mortelle de plusieurs dizaines de sievert. Après une période de 1000 ans, la même durée d'exposition à proximité d'un colis donnerait lieu à une dose de quelques millisievert. La figure 2 présente l'évolution du débit de dose $\beta \gamma$ en sievert par heure pour un colis et à une distance d'un mètre en latéral du colis (Lagrange, 2005).

\subsubsection{Incertitudes}

Les stockages de déchets prévus en formation géologique sont généralement situés dans des environnements dont les caractéristiques clefs pour la sûreté de l'installation (stabilité mécanique, hydrogéologie, ...) sont peu susceptibles d'être modifiées au cours du temps. Cependant, au regard des échelles de temps considérées pour les déchets, même l'environnement le plus stable peut être sujet à des perturbations. L'incertitude sur ces perturbations augmente avec le temps et est prise en compte dans les études de sûreté. Dans ce contexte, bien que la démarche de sûreté ait pour objectif d'analyser dans quelle mesure les dispositifs de protection apportent le maximum de garanties quant aux évolutions possibles sur le long terme, des degrés d'incertitudes subsistent inévitablement. 


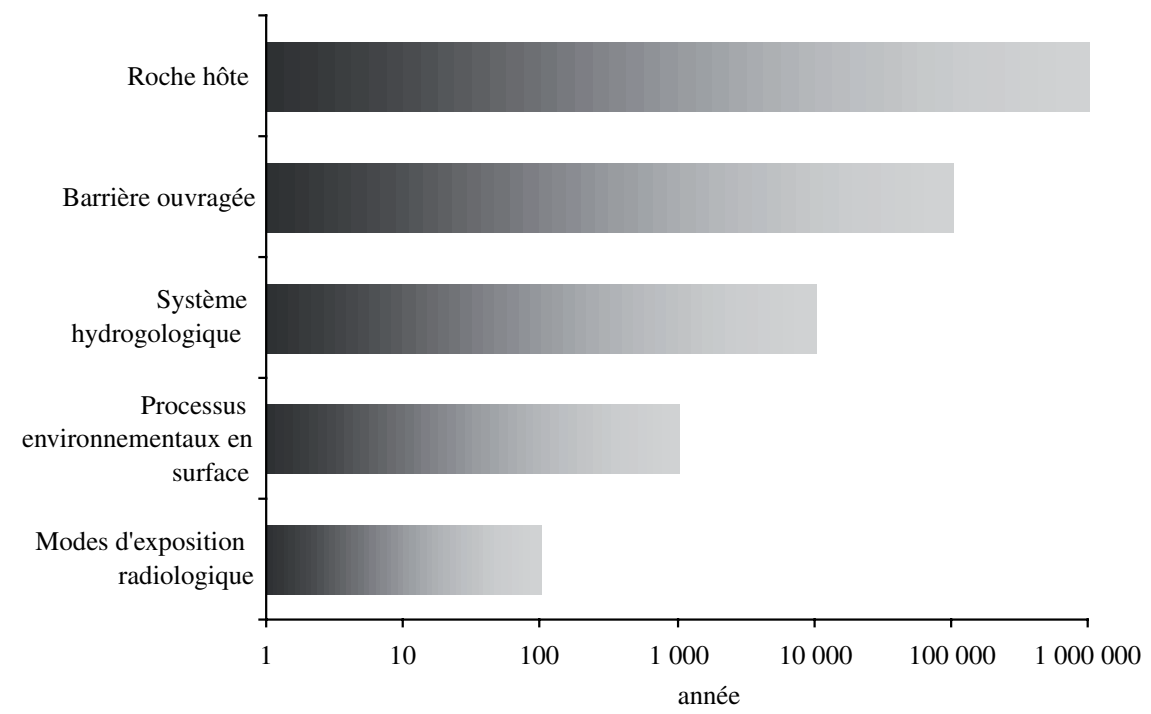

Figure 3 - Les limites de prévisibilité des divers aspects du système de stockage en formation géologique. The limits of predictability of various aspects of a geological disposal system.

À titre d'illustration, on peut ainsi citer un rapport de l'Agence pour l'énergie nucléaire (AEN) de l'OCDE (AEN/OCDE, 2004) qui souligne les limites de la prédictabilité de divers composants d'un système de stockage géologique (voir Fig. 3) : «Il apparaît que, pour un site bien choisi, l'évolution des caractéristiques générales des barrières ouvragées et de la roche hôte est raisonnablement prévisible sur une longue période (disons $10^{5}$ ou $10^{6}$ années, par exemple, dans le cas de la roche hôte). [...] Les caractéristiques de la circulation de l'eau souterraine (système hydrogéologique), en particulier près de la surface, dépendent du changement climatique et, de ce fait, sont moins prévisibles (de l'ordre de $10^{4}, 10^{5}$ ans). Les processus environnementaux à la surface et les modes d'exposition radiologiques ne sont en général pas considérés comme faisant partie du système de stockage géologique mais doivent être pris en compte pour évaluer la dose et le risque. Ceux-ci sont encore moins prévisibles étant donné qu'ils dépendent de l'évolution de l'environnement, des activités humaines et des habitudes individuelles qui sont incertaines même à une échelle de quelques années ». Face à ces incertitudes, les critères et les principes adoptés par les autorités pour l'évaluation de la sûreté des systèmes de stockage géologique sont généralement séparés en deux périodes : une période de l'ordre de 10000 ans pendant laquelle la prédictabilité de la performance des systèmes est considérée comme accessible et est basée sur les évaluations des paramètres cités ci-dessus, 
PRISE EN COMPTE DU LONG TERME ET MODALITÉS DE GESTION DES DÉCHETS RADIOACTIFS

et une période au-delà de 10000 ans où les évaluations sont complétées par des appréciations plus qualitatives des résultats au regard des facteurs d'évolution.

\subsection{Le long terme du point de vue sociétal}

Les éléments présentés ci-dessus montrent clairement que les échelles de temps utilisées dans les évaluations techniques de sûreté des stockages de déchets radioactifs se situent hors du champ habituellement considéré pour les prévisions d'évolution de la société. La prise en compte du long terme dans les modalités de gestion des déchets ne peut cependant pas être limitée aux considérations techniques. Étant donné les risques potentiels associés aux déchets radioactifs, il est essentiel de reconnaître que c'est la société dans son ensemble qui est concernée par le problème, et non pas seulement les producteurs de déchets, les institutions en charge de leur gestion ou les autorités. Bien que la responsabilité de la gestion des déchets repose sur ces différents acteurs, c'est la société toute entière qui s'engage dans un processus de gestion de long terme des déchets, ce qui introduit de nouvelles responsabilités envers les générations futures : poursuivre la gestion, maintenir et organiser la surveillance et la conservation de la mémoire des installations de gestion des déchets.

Dans les premiers temps, les réflexions éthiques menées dans le cadre de la gestion des déchets radioactifs ont conduit à introduire l'obligation pour la société actuelle d'éviter de faire supporter des « charges indues » aux générations futures. Bien que le devoir de protéger les générations futures soit de prime importance, la capacité à réellement remplir cette obligation dépend très largement des incertitudes techniques et scientifiques, ainsi que de l'évolution de la société. Dans ce contexte, il apparaît que les générations futures seront toujours confrontées aux risques, même si ces derniers restent potentiels au cours du temps. De plus, il convient de s'interroger sur le droit que s'accorde la génération actuelle à imposer un comportement aux générations futures, tout en reconnaissant que ce comportement est dicté par des objectifs de protection. Dans cette perspective, il apparaît qu'une approche raisonnable pour prendre en compte cette préoccupation consiste, pour la génération actuelle, à créer des processus favorisant une transmission continue vers la (les) future(s) génération(s) d'un «patrimoine de sécurité » (savoir-faire, options de protection, procédures, ressources, ...) afin d'assurer la continuité de la gestion des déchets. Les générations futures pourront faire évoluer ces processus, mais il importe que la génération actuelle considère dès maintenant comment ils peuvent être élaborés, et notamment, quelles sont les « missions » qui devront être transmises aux générations futures. Celles-ci auront ensuite la responsabilité de continuer et/ou de reconsidérer ces processus et de les adapter en fonction des évolutions tant sociales que techniques, voire environnementales. 
Cette approche implique de composer avec l'organisation passée, présente et future de la gestion des déchets radioactifs et d'introduire un processus permettant aux générations futures d'intervenir. On peut noter que dans cette perspective, le concept de réversibilité introduit de la flexibilité dans le processus de décision. Néanmoins, il convient de garder en mémoire le fait qu'être flexible ne signifie pas reporter les décisions, mais construire un dispositif de protection intégrant une capacité d'action pour les générations futures.

\subsection{Vers une complémentarité des approches}

Quelles que soient les options techniques qui seront adoptées, il est nécessaire de combiner les deux préoccupations principales pour le long terme (c'est-à-dire technique et sociétale). Du point de vue de la sûreté et de la radioprotection, une efficacité absolue sur les échelles de temps considérées ne peut pas être démontrée. C'est uniquement par un transfert de responsabilité entre les générations que la gestion des déchets peut trouver un chemin. Par conséquent, la génération actuelle doit s'interroger sur l'efficacité et la faisabilité des options techniques, en association avec un système de gestion permettant de répondre à la demande sociétale en termes de transmission $d u$ «patrimoine de sécurité ».

\section{Enjeux éthiques associés à la dimension de long terme de la gestion des déchets radioactifs}

Les dimensions éthiques, organisationnelles et politiques ont déjà été étudiées par les experts de la gestion des déchets radioactifs dans diverses organisations : l'Agence internationale de l'énergie atomique (AIEA, 1992), l'Agence de l'énergie nucléaire de l'OCDE (AEN/OCDE, 1995), le comité consultatif suédois sur la gestion des déchets radioactifs (KASAM-SKN, 1988), la Commission Seaborn au Canada, etc. Comme noté précédemment, l'une des principales conclusions de ces experts est de considérer que le principe directeur pour l'élaboration des options de gestion des déchets est d'éviter les «charges indues » pour les générations futures. Dans le projet COWAM 2, la perspective adoptée a consisté à rechercher comment créer les meilleures conditions pour favoriser le transfert de l'ensemble du système de gestion des déchets à la prochaine génération et aux suivantes. Ceci a conduit à l'identification de trois principes éthiques majeurs comme étant des points clefs de la prise en compte du long terme dans les modalités de gestion des déchets radioactifs : la responsabilité, la justice et la démocratie.

La responsabilité stipule qu'un individu, ou un groupe dans le cas de la responsabilité collective, est capable de répondre de ses actes, et par extension, de les assumer, y compris s'il n'y avait pas au départ l'intention de produire certains 
effets. La responsabilité de long terme, notamment dans le cas des déchets radioactifs, suggère qu'il existe une asymétrie entre générations, et qu'il n'y a donc pas de réciprocité entre elles. En effet, la génération $n$ ne sera plus en vie à l'époque de la génération $n+10$, par exemple, même si par ailleurs ses actions auront des conséquences dans le long terme. Cependant, l'absence de réciprocité entre générations n'est pas une raison pour écarter toute responsabilité envers le futur, et, au contraire, elle enjoint de créer de nouvelles formes de responsabilité (inter et trans-générationnelle).

La justice concerne l'évaluation des actions sur la base d'un principe d'égalité ou d'équité (proportionnalité) dans le cadre de la relation qu'un individu ou un groupe entretient avec l'ensemble de la communauté. La question de la justice de long terme requiert de la génération $n$, responsable de l'augmentation du volume de déchets, qu'elle apporte une contribution proportionnée aux générations futures qui seront affectées par les déchets. Il peut donc y avoir une justice entre générations, une justice inter-générationnelle, dès lors que sont prévues les modalités de la contribution que la génération n pourra apporter à la génération $n+10$, par exemple.

Enfin, la démocratie désigne le régime politique dans lequel la légitimité des actions dépend de la représentation et de la participation de la population aux processus de délibération et de décision collective.

L'analyse de ces principes a permis au groupe de travail d'élaborer ensemble 20 critères éthiques susceptibles d'être utilisés pour l'évaluation des modalités de gestion des déchets radioactifs. Ces critères sont présentés ci-après.

\subsection{La responsabilité de long terme}

"Les générations futures devraient recevoir des moyens durables appropriés (en termes de processus, finances, institutions, connaissances, savoir-faire, ...) pour la mise en auvre et l'évaluation des systèmes de gestion des déchets. »

«Ces moyens durables devraient être conçus en particulier pour garantir la protection à long terme de la santé et de l'environnement. »

«La distribution des responsabilités entre le secteur public et le secteur privé dans le domaine de la protection de la santé et de l'environnement devrait être clairement établie, ainsi que son évolution au cours du temps. »

«La conservation de l'information, des connaissances et des savoir-faire concernant la gestion des déchets radioactifs devrait être mise en ouvre par des mesures, des organisations et des réseaux appropriés. » 
«Ces éléments devraient être durables et disponibles pour les acteurs ainsi que pour l'éducation des générations futures. »

"Une politique responsable de gestion de long terme des déchets radioactifs devrait articuler de façon flexible les décisions actuelles avec les possibilités d'action futures.

\subsection{La justice de long terme}

"L'équité d'un système de gestion des déchets devrait être évaluée en termes d'avantages et d'inconvénients, sur la base des relations intra / inter / transgénérationnelles. 》

"Cette évaluation devrait intégrer les aspects quantitatifs et qualitatifs des conditions de vie, ainsi que les contextes et tendances économiques, sociales et techniques les plus probables. »

"Une génération responsable de la création des déchets devrait fournir une contribution aux personnes affectées par ces déchets, qu'elles appartiennent à la même génération ou aux générations futures. »

"Cette contribution devrait être proportionnée aux efforts consentis (notamment du point de vue de la recherche et du développement) en matière de gestion des déchets radioactifs et d'optimisation du coût des systèmes de gestion. »

"Une municipalité qui accepte de gérer les déchets radioactifs du pays devrait bénéficier d'une solidarité de long terme de la part de la nation. »

"Une municipalité qui accepte une installation de gestion des déchets radioactifs devrait recevoir un financement pour le développement socioéconomique du territoire.

"Ce financement devrait être destiné à soutenir un développement durable du territoire afin d'assurer la continuité de la vigilance et de la surveillance du site par la participation de la population locale.

\subsection{La démocratie de long terme}

«Un système de gouvernance démocratique de long terme nécessite la mise en place d'une organisation politique flexible combinant la représentation, la participation et la délibération des citoyens. » 
"La gouvernance à long terme des déchets radioactifs devrait créer un lien entre les options techniques et la démocratie participative.»

"L'institution en charge de la gestion des déchets radioactifs devrait être soumise à un contrôle démocratique et être contre-balancée par une implication des citoyens à travers les générations.

"Une organisation en charge de la continuité de la participation, de la pluralité de l'expertise, de l'information, des conceptions et des valeurs devrait pouvoir conserver une indépendance, notamment financière, à l'égard des autorités techniques et politiques et des associations civiles. »

«Un processus de gouvernance démocratique devrait rassembler une grande diversité de personnes, de plusieurs générations, et d'origines différentes (local 1 national / international, autorités / experts / citoyens / associations / exploitants...). »

«Afin de rester un sujet de débat au cours du temps, le thème des déchets radioactifs devrait être inscrit régulièrement sur l'agenda politique des organisations gouvernementales ou non-gouvernementales, que ce soit au niveau local, national ou international. »

"Tous les citoyens devraient pouvoir disposer des moyens et des informations nécessaires pour participer pleinement au processus et exercer leurs droits. »

\section{Continuité et durabilité de la surveillance et du contrôle}

Quel que soit le type d'installation de gestion des déchets (stockage géologique, entreposage de court terme ou pérennisé), le terme générique de «surveillance » peut comporter différents aspects des systèmes de protection, qui peuvent également varier dans le temps, tels que :

- la surveillance du site ;

- le contrôle de l'environnement de l'installation, la maintenance de l'installation, la gestion de toutes les activités sur le site, y compris la récupérabilité des colis de déchets ;

- la préservation et la transmission des savoir-faire concernant la gestion des déchets ;

- la formation des générations qui assureront le relais ;

- l'organisation d'une vigilance à différents niveaux.

La continuité et la durabilité de la surveillance et du contrôle sur le long terme ne peuvent être ni garanties, ni décrétées. Il n'est pas non plus possible de définir maintenant comment la société devra être organisée demain pour assurer la gestion 
des déchets. Dans un processus de gouvernance du long terme, il convient dès lors de rechercher comment créer les conditions pour favoriser la préservation de la vigilance (aux niveaux local, national et international) ainsi que son transfert à travers les générations.

Afin d'étudier les points clefs associés à la continuité et à la durabilité des systèmes de protection, deux études de cas ont été réalisées : les systèmes mis en place par l'UNESCO pour la protection, la préservation et la transmission aux générations futures des sites inscrits au Patrimoine Mondial de l'humanité et la gestion des anciennes mines de fer de Lorraine en France (Hériard-Dubreuil et al., 2003). Elles ont été complétées par des réflexions sur l'intégration des systèmes de protection dans des projets de développement socio-économiques durables des territoires. Ce sujet a notamment bénéficié des propositions de parties prenantes : (i) celles du groupe de parties prenantes locales - MONA - qui a été créé dans la municipalité de Mol (Belgique) pour discuter de la faisabilité d'un stockage de déchets radioactifs de faible et moyenne activités avec l'ONDRAF/NIRAS (Organisme National des Déchets Radioactifs) (Meus et Ceulemans, 2003 ; MONA, 2005) ; (ii) les propositions formulées lors du débat public sur la gestion des déchets radioactifs qui s'est tenu en France de septembre 2005 à janvier 2006 (Schneider, 2005).

L'ensemble de ces analyses ont permis d'identifier plusieurs champs d'actions qui pourraient être étudiés lors de l'élaboration des systèmes de surveillance et de contrôle autour des installations de gestion des déchets radioactifs afin de favoriser la durabilité de ces systèmes sur le long terme. Les principaux éléments à considérer au sein de ces champs d'actions sont présentés ci-dessous.

\subsection{L'organisation de la surveillance et de la vigilance}

- La transmission entre générations du système de surveillance devrait être étudiée pour favoriser une conservation active de la mémoire de l'installation. À cette fin, il est notamment nécessaire de permettre une évolution du système de gestion des déchets et de surveillance des installations avec le temps.

- Les parties prenantes locales devraient être impliquées dans le système de surveillance de l'installation. Elles représentent en effet des acteurs clefs de la vigilance et de la transmission entre générations.

- Le programme de surveillance et de contrôle devrait être clairement organisé (répartition des responsabilités, procédures de contrôle, ....). La durabilité d'un tel programme serait notamment renforcée par la création de points de rendezvous réguliers avec les organismes de contrôle de l'État, l'organisme en charge de la surveillance, et les acteurs locaux, afin d'évaluer son efficacité et d'identifier les besoins d'évolution. 
- Il conviendrait également d'associer au programme de surveillance, un système financier dédié et durable. La capacité à mobiliser, si nécessaire, des ressources internationales devrait aussi être étudiée.

\subsection{Le développement d'un pôle de compétences}

- Un pôle de compétences pourrait être créé pour assurer l'exploitation, la maintenance et la surveillance de l'installation de gestion des déchets sur le long terme.

- Les objectifs de ce pôle de compétences devraient être focalisés sur le développement, l'utilisation et la transmission aux générations suivantes de l'expertise et des savoir-faire pour assurer une surveillance et un contrôle efficaces de l'installation dans le temps.

- Le pôle de compétences devrait pouvoir bénéficier de l'expertise locale, nationale et internationale. Les possibilités d'utiliser l'expertise de ce pôle en différents endroits et dans d'autres domaines que la gestion des déchets radioactifs devraient être favorisées.

- L'implication de parties prenantes dans la définition et le suivi des activités du pôle de compétences est également un élément important de sa durabilité et du maintien de la vigilance sur le long terme.

\subsection{Intégration de l'installation de gestion des déchets radioactifs et de sa surveillance dans un développement socio-économique local/régional}

- La fonction de surveillance devrait pouvoir être intégrée dans un projet global de développement socio-économique durable des territoires. Un tel projet devrait notamment être élaboré en vue de maintenir «la vie» autour de l'installation de gestion des déchets radioactifs, car la stabilité de la démographie locale et régionale constitue un élément clef pour la durabilité de la surveillance.

- Il conviendrait, par exemple, d'étudier le développement d'activités économiques associées à la surveillance et au contrôle de l'environnement, en interaction avec les compétences scientifiques et technologiques au niveau local et régional.

- Il est également essentiel de mettre en place des systèmes permettant de s'assurer que la présence de l'installation de gestion des déchets radioactifs est compatible avec le développement du territoire sur le long terme. 


\subsection{Une distribution équitable des responsabilités entre les territoires et les générations}

- Un système de protection efficace nécessite une distribution claire des responsabilités entre les acteurs locaux, nationaux et internationaux.

- La notion de « patrimoine de sécurité » devrait être développée afin de créer un «lien de sécurité » entre les acteurs locaux, nationaux et internationaux, et entre les générations.

- Il pourrait enfin être envisagé d'engager des réflexions sur l'intérêt d'une convention internationale sur la protection des installations de gestion des déchets radioactifs.

\section{Efficacité des mécanismes de financement pour la gestion des déchets radioactifs sur le long terme}

La capacité des générations futures à mettre en œuvre des options de gestion des déchets radioactifs et à poursuivre le contrôle et la surveillance repose notamment sur les ressources financières qui seront disponibles dans le futur. Afin d'identifier les principales caractéristiques des mécanismes financiers et de proposer quelques questions relatives à leur durabilité sur le long terme, les mécanismes mis en place dans quelques pays européens (Allemagne, Belgique, Espagne, Finlande, France, Suède, Suisse) ont été analysés en se focalisant plus précisément sur les éléments suivants :

- la distribution des responsabilités entre les différents acteurs (producteurs de déchets, organisme en charge de la gestion des déchets, État, ...);

- le type de mécanisme financier et le cadre juridique. Deux types de mécanismes principaux ont été mis en évidence : ceux basés sur la gestion d'un fonds dédié, alimenté par les producteurs de déchets, mais géré soit par l'organisme de gestion des déchets, soit par un organisme national indépendant ; ceux basés sur une gestion par les exploitants de provisions et d'actifs dédiés sur leur propre compte ;

- les processus de décision sur la gestion des fonds et leur réévaluation dans le temps ;

- les mécanismes de garanties financières.

L'analyse de ces différents mécanismes financiers, couplée à une réflexion sur les éléments de leur durabilité a permis d'identifier quelques éléments qui apparaissent essentiels à étudier pour évaluer la performance de tels mécanismes dans une perspective de long terme. 


\subsection{La distribution des responsabilités concernant la gestion des déchets radioactifs}

Ces responsabilités comprennent la propriété des déchets, la responsabilité pour le financement, pour la mise en service de l'installation de gestion des déchets, pour la surveillance, ... Le transfert de ces responsabilités au cours du temps devrait être prévu à l'avance.

\subsection{La transparence autour des estimations de coût et d'utilisation des fonds}

- Le processus de décision pour définir le montant des fonds ou des provisions ainsi que leur utilisation devraient être expliqués, ainsi que les scénarios de gestion des déchets radioactifs utilisés pour déterminer les besoins financiers dans le futur.

- En particulier, il semble nécessaire de considérer les coûts associés à la surveillance de long terme ou à l'accompagnement financier pour le développement durable des territoires.

- Il conviendrait également de clarifier les mécanismes permettant de faire évoluer les fonds dans le temps. Un contrôle externe des fonds ou des provisions devrait être réalisé périodiquement par l'État en collaboration avec des parties prenantes au niveau local et national, par exemple en impliquant la commission locale d'information dans le suivi de la gestion des fonds.

\subsection{Garanties financières}

- Les mécanismes financiers devraient intégrer des garanties financières au cas où le coût de la gestion des déchets radioactifs serait plus élevé que prévu ou en cas de faillite d'un producteur de déchets.

- Ils devraient également intégrer des systèmes spécifiques pour s'assurer (autant que possible) que l'argent provisionné sera disponible au moment où il sera nécessaire.

\section{Conclusion : quels enjeux pour la radioprotection ?}

La prise en compte du long terme dans les modalités de gestion des déchets radioactifs nécessite de combiner des considérations techniques et des dimensions éthiques, économiques, politiques et organisationnelles. Ces dimensions sont des éléments essentiels pour permettre à la société de s'engager dans un système de protection sur le long terme. L'objectif d'un tel système n'est pas, bien entendu, 
de définir comment les sociétés de demain devront être organisées pour gérer les déchets, mais bien de mettre en place des dispositions favorisant une transmission de l'ensemble du système de protection à la (aux) génération(s) à venir. Ainsi, les performances d'une installation de gestion des déchets radioactifs (entreposage ou stockage) devraient être évaluées en considérant un système complet de protection intégrant bien sûr des dimensions techniques mais aussi organisationnelles relatives à la transmission des connaissances et des savoir-faire, à l'organisation de la surveillance et son évolution au cours du temps, à l'intégration de l'installation dans un projet de développement socio-économique durable au niveau territorial, etc.

À cette fin, il apparaît essentiel que la conception des options de gestion des déchets radioactifs ne soit pas seulement l'affaire des experts scientifiques et techniques, mais qu'elle implique également d'autres acteurs de la société qui seront concernés directement ou indirectement par l'existence de ces installations de gestion des déchets et le maintien de leur efficacité et de la mémoire de ces installations dans le temps. Il est donc important d'élaborer des modalités de coordination sur le long terme (points de rendez-vous, lieux de dialogue, identification des responsabilités de chacun, ...) entre les différents acteurs (autorités, experts, citoyens, associations, exploitants, ...), basées sur une implication à plusieurs niveaux (local, national et international).

Dans cette perspective, il est intéressant de noter qu'en France, le débat public sur la gestion des déchets et la loi n 2006-739 du 28 juin 2006 relative à la gestion durable des matières et des déchets radioactifs ont apporté des éléments d'ordre non seulement technique, mais aussi économique, sociétal ou organisationnel dans les dispositifs de gestion des déchets radioactifs.

Pour les professionnels de la radioprotection, l'élaboration et la mise en œuvre de systèmes de protection pour les déchets radioactifs répondant aux enjeux techniques et sociétaux de long terme constituent un challenge pour les années à venir. Il s'agit à la fois de favoriser le développement d'une culture de radioprotection parmi les différents acteurs afin de permettre un véritable dialogue sur les enjeux associés à la protection sur le long terme, mais également de s'interroger sur les modalités pratiques concernant l'évaluation de la performance du système de protection au cours du temps, l'organisation de la vigilance et de la surveillance, les conditions de transfert et de maintien des connaissances en radioprotection pour les générations futures. 


\section{Encart}

Une grille d'analyse pour identifier les enjeux de long terme dans un dispositif de gestion des déchets radioactifs

Les recherches menées dans le cadre du groupe de travail du projet COWAM ont permis de proposer un ensemble de critères éthiques spécifiques à la prise en compte de la dimension du long terme ainsi que des réflexions concernant les dispositions institutionnelles, financières et sociétales favorisant la durabilité des systèmes de gestion des déchets radioactifs.

Afin de faciliter l'identification des enjeux de long terme dans les dispositifs de gestion des déchets radioactifs, une grille d'analyse est proposée (Tab. I). Cette grille relie les processus techniques aux enjeux éthiques par un ensemble de conditions institutionnelles, financières et sociétales. Elle contient les principaux éléments qu'il convient de considérer lorsque l'on s'interroge sur la robustesse d'un dispositif de gestion des déchets par rapport à la prise en compte long terme.

Cette grille est destinée à favoriser un dialogue entre les différents acteurs de la gestion des déchets (au niveau local, national ou international) en vue d'une évaluation commune des dispositifs de gestion des déchets radioactifs sur le long terme destinée à identifier les points forts de ces dispositifs ainsi que les voies d'amélioration possibles.

Remerciements. Le projet COWAM 2 a été co-financé par la Commission européenne dans le cadre du programme Euratom de recherche et de formation sur l'énergie nucléaire au sein du sixième programme-cadre de recherche (20022006). Contrat numéro : FI6W-CT-2003-508856. Le CEPN a également bénéficié d'un soutien de l'IRSN pour ce projet.

Annexe 1 - Le groupe de travail sur la prise en compte du long terme dans les modalités de gestion des déchets radioactifs du projet européen COWAM 2

Le projet européen COWAM 2 sur la gouvernance de la gestion des déchets radioactifs, coordonné par Mutadis Consultants, s'est déroulé de 2004 à 2006. Il avait pour objectif de contribuer à l'amélioration de la qualité des processus de décision dans la gestion des déchets nucléaires. Le programme de recherche a été articulé autour de quatre groupes de travail thématiques composés de parties 
C. SCHIEBER et al.

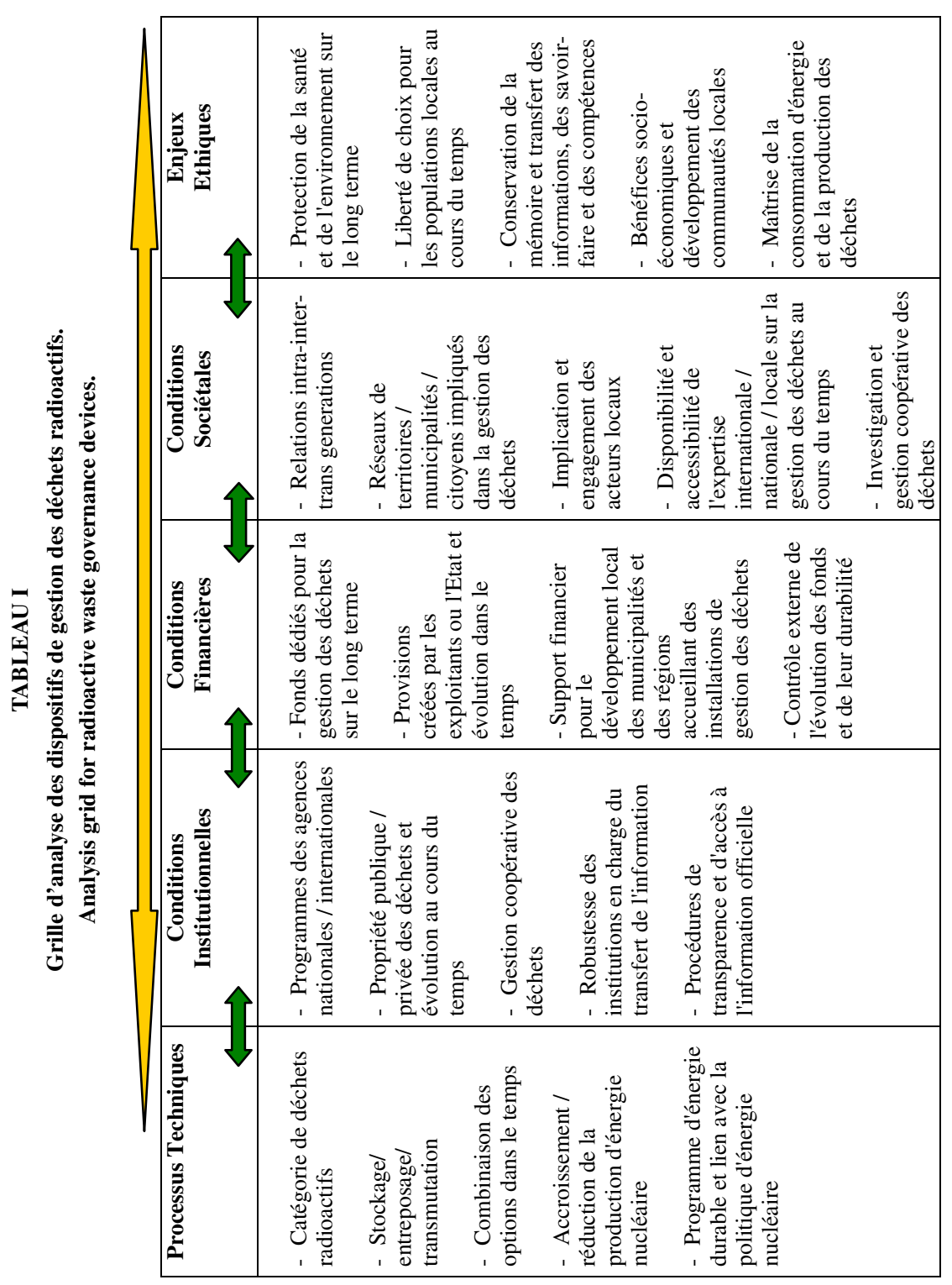


prenantes de différents pays d'Europe et d'une équipe de recherche ${ }^{6}$. Les thèmes de ces groupes étaient les suivants :

- les pratiques de démocratie locale et les méthodes d'évaluation participatives,

- l'influence des acteurs locaux sur les processus de décisions nationaux,

- la qualité des processus de décisions associés à la recherche de sites,

- la prise en compte du long terme dans les modalités de gestion des déchets radioactifs.

Les réunions du groupe de travail dédié à la prise en compte du long terme ont rassemblé deux fois par an pendant les trois ans du projet COWAM 2, une vingtaine de participants : membres des commissions locales d'information et des associations, des exploitants d'installations nucléaires et de gestion des déchets, des régulateurs ainsi que des organismes public d'expertise ou de recherche. Ils provenaient d'Allemagne, de Belgique, d'Espagne, de France, des Pays-bas, de Roumanie, du Royaume-Uni, de Suède et de Suisse. L'équipe de recherche était composée de quatre instituts de Belgique, de France et de Suisse, et comprenait des experts dans le domaine de l'éthique, de la radioprotection, de l'économie, de l'évaluation environnementale et des sciences sociales.

L'approche adoptée s'est structurée autour des étapes suivantes :

- identification des sujets à développer,

- préparation par l'équipe de recherche de documents de travail spécifiques ( « définition » du long terme, considérations éthiques, ...) et d'études de cas (durabilité des systèmes de protection, dispositifs financiers de long terme, ...) pour engager des discussions lors des réunions du groupe de travail,

- contribution des parties prenantes (réflexion sur l'éthique, les contextes locaux et nationaux, les mécanismes financiers pour prendre en compte le long terme dans les modalités de gestion des déchets, ...),

- préparation d'un projet de rapport final par l'équipe de recherche,

- commentaires et validation de ce rapport par les participants (y compris lors d'une réunion spécifique).

Il importe de mentionner qu'au début du projet, plusieurs participants ont notifié que, de leur point de vue, le préalable à leur implication dans la gestion des déchets radioactifs était d'aborder la question de l'articulation entre les scénarios de politique énergétique conjointement avec les scénarios de gestion des déchets. Bien que ce problème ait été considéré comme important, l'ensemble des participants ont reconnu que COWAM 2 n'était pas l'endroit où le traiter, mais qu'il nécessitait un forum adéquat au niveau national, intégrant l'ensemble des

6 Les résultats de COWAM 2 sont disponibles sur le site Internet : www.cowam.com 
parties prenantes pertinentes. Il a par contre été convenu de mentionner clairement cette remarque dans toute publication relative aux travaux du groupe de travail.

Annexe 2 - Les membres du groupe de travail sur la prise en compte du long terme dans les modalités de gestion des déchets radioactifs

\section{Équipe de recherche}

France : Thierry Schneider (Coordinateur) et Caroline Schieber (CEPN) ; Sylvain Lavelle (ICAM) ;

Belgique : Michel Bovy, Gunter Bombaerts et Gaston Meskens (SCK-CEN Mol); Suisse : Thomas Flüeler (ETH Zurich).

\section{Parties prenantes}

Belgique : Hugo Ceulemans, Jacques Helsen et Joss Prost (MONA-Mol) ;

Allemagne : Eckhard Kruse (Gartow Church representative) ; Jürgen Wollrath (BfS) ;

Europe : Laurent Furedi et Mark O'donovan (FORATOM, Europe) ;

France : Geneviève Baumont (IRSN) ; Eric Chagneau (GIP Objectif Meuse) ; Joël Chupeau (EDF) ; Robert Granier (CLI du Gard) ; Benoit Jaquet et Jérôme Sterpenich (CLIS de Bure) ; Olivier Laffitte (CSPI La Hague) ; Alain Marvy (CEA) ; Chantal Rigal (ANCLI ) ; Wolf Seidler (ANDRA - projet ESDRED) ;

Pay-Bas : Herman Damveld (chercheur indépendant);

Roumanie : Stela Diaconu (ANDRAD);

Espagne : Felisa García (ENRESA) ; Miquel Ferrús Serar (GMF) ; Fernando García (Maire de Jarafuel) ; Jose Luis Gómez (Maire de Frias) ; M. Hernández (Maire de Almaraz) ; Meritxell Martel (ENVIROS ) ; Alfredo Navaro (Maire de Valencia) ; Alfredo Romero (Maire de Mesas de Ibor) ;

Suède : Olov Holmstrand (Avfallskedjan - The Waste Network);

Suisse : Pius Krütli (ETH Zurich);

Royaume-Uni : Shelly Mobbs (HPA).

\section{RÉFÉRENCES}

AEN/OCDE (1995) Agence pour l'énergie nucléaire/Organisation de coopération et de développement économiques, La gestion des déchets radioactifs à vie longue : fondements environnementaux et éthiques de l'évacuation géologique, opinion collective du Comité de la gestion des déchets radioactifs de l'AEN, OCDE. 
PRISE EN COMPTE DU LONG TERME ET MODALITÉS DE GESTION DES DÉCHETS RADIOACTIFS

AEN/OCDE (2004) Agence pour l'énergie nucléaire/Organisation de coopération et de développement économiques, Gestion des échelles de temps dans l'évaluation de la sûreté en phase postfermeture Enseignements tirés de l'atelier d'avril 2002 à Paris, France, NEA nº 5148.

AIEA (1992) Agence internationale de l'énergie atomique, Radiaoctive Waste Management, an IAEA Source Book, Vienna.

Hériard-Dubreuil G., Schieber C., Devillers C., Delaigue S., Schneider T. (1996) Les enjeux sociaux de la surveillance institutionnelle des stockages profonds de déchets radioactifs, Rapport CEPN R-248.

Hériard-Dubreuil G., Schieber C., Schneider T. (1998) Les enjeux sociaux de la réversibilité dans le stockage profond des déchets radioactifs de haute activité, Rapport CEPN R-258.

Hériard-Dubreuil G., Gadbois S., Schieber C., Schneider T. (2003) Réflexion sur les critères de performance d'un entreposage de longue durée (ELD) vis-à-vis des situations de délaissement temporaire, Rapport CEA - DEN/DDIN/DPRGD/2003/3.

Hériard-Dubreuil G. Gadbois S., Mays C., Espejo R., Flüeler T., Schneider T., Paixa A. (2007) COWAM 2 - Cooperative Research on the Governance of Radioactive Waste Management, Final Synthesis Report, June 2007, Mutadis, Paris, EC contract FI6W-CT-2003-508856.

KASAM-SKN (1988) Ethical Aspects on Nuclear Waste - Some Salient Points Discussed at a Seminar on Ethical Action in the Face of Uncertainty in Stockholm, Sweden, September 8-9, 1987, SKN report 29.

Lagrange M.H. (2005) Modèle d'inventaire de dimensionnement (MID). données descriptives du colis type C1. Projet HAVL, Note technique ANDRA C.NT.AHVL.02.109, Indice B, Septembre 2005.

Lavelle S. (2006) Science, technologie et éthique - Conflits de rationalité et discussion démocratique. Ellipses Edition, Collection Technosup.

Meus B., Ceulemans H. (2003) MONA, Public Participation in the Siting of a LLW Repository in Mol, Belgium, in Proceedings of ICEM'03, September 21-25, Oxford, England.

MONA (2005) Mols Overleg Nucleair Afval Categorie A: Een weg naar de aanvaardbaarheid van een berging van categorie A-afval in Mol? Geïntegreerd eindrapport.

Schneider T. (2005) Éléments de réflexion sur les devoirs pour la génération présente pour le gardiennage à long terme des déchets radioactifs, Contribution du CEPN à la réunion du débat public organisée à Nancy le 18 novembre 2005 par la Commission Particulière du Débat Public sur la gestion des déchets sur le thème: Démocratie et déchets radioactifs: Juste équilibre entre générations et territoires, Note CEPN NTE/05/39.

Schneider T., Schieber C., Lavelle S. (2006) Long Term Governance for Radioactive Waste Management, Final Report of COWAM2, Work Package 4, Report COWAM2-D4-12 / CEPNR-301. 